\title{
An LMI-Based Optimization Approach for Integrated Plant/Output-Feedback Controller Design
}

\author{
Fang Liao, Kai Yew Lum and Jian Liang Wang
}

\begin{abstract}
In this paper, a special integrated plant/output-feedback controller optimization problem is studied, which optimizes a single plant parameter while satisfying open- and closed-loop static and dynamic performance requirements. Both polytopic model uncertainties and multi-mission optimization are considered in the integrated design optimization. A necessary and sufficient condition is given for the solvability of such an optimization problem. Based on the condition, a design optimization method is presented in terms of linear matrix inequalities. An aircraft example is applied to demonstrate the proposed method.
\end{abstract}

Keywords: integrated plant/controller design, optimization, linear matrix inequalities(LMIs)

\section{Introduction}

Traditionally, the design optimization of a plant, such as aircraft and underwater vehicles, and its controller abides a sequential strategy whereby the plant is designed and optimized first, followed by the controller. Although this sequential strategy has been used successfully in most of the missions in the past, it may not guarantee system-level optimality as the plant and controller optimization problems are coupled. Such coupling is widely accepted in the control community [3], but is minimized in the sequential strategy.

It has been recognized that significant improvements in the overall system performance and cost are possible if the process of plant design and control system development were integrated. This integrated design strategy is to simultaneously optimize the design parameters of both the plant and controller so as to satisfy desired design specifications and optimize the closedloop performance. Recent research on flexible space structures [6], aeroservoelasticity [8], mechatronics [11], electric motors, and many other systems has shown that the integrated strategy furnishes better systems than the sequential strategy. Nevertheless, the integrated plant/controller optimization problem is not guaranteed to be convex even when the plant and controller optimization subproblems are individually convex. This

\footnotetext{
This work is supported under grant DSTA POD 1820, Singapore.

F. Liao is with Temasek Laboratories, National University of Singapore, Singapore 117508. Email: tsllf@nus.edu.sg.

K. Y. Lum is with Temasek Laboratories, National University of Singapore, Singapore 117508. Email: tsllumky@nus.edu.sg.

J. L. Wang is with School of Electrical and Electronic Engineering, Nanyang Technological University, Singapore 639798. Email: ejlwang@ntu.edu.sg.
}

makes the integrated strategy mathematically and computationally challenging.

Recently, several integrated $H_{\infty}$ plant/controller design approaches have been presented [7], [4], [10]. In [7], an integrated aircraft/controller design is formulated and an iterative LMI-based algorithm is suggested to solve the optimization problem: at each iteration, for a fixed plant find a feasible controller, and then for a fixed controller minimize the cost function over the plant parameters. However, only state-feedback is considered and convergence properties cannot be guaranteed for the proposed algorithm. In [10], Yang and Lum continue to pursue the integrated plant/state-feedback controller design optimization problem. An iterative LMI-based algorithm with convergence properties is proposed. The dynamic output-feedback problem is considered by Grigoriadis and Wu [4], also using an iterative LMI approach. The proposed algorithm guarantees convergence. Unfortunately, the assumption that the control input matrix is independent of design parameters of the plant seems too restrictive. Moreover, all of these approaches did not consider model uncertainties and multi-mission operation.

This paper investigates a special type of integrated plant/output-feedback controller optimization problems where only one plant parameter is to be optimized, e.g., designing the size of a control surface (such as an elevator) of an aircraft. As is well-known, a smaller elevator results in a low overall aircraft system cost and low drag but a downgraded overall aircraft system performance. On the contrary, a larger elevator results in a high overall system cost and high drag but a better overall system performance. Hence, there is a trade-off between the size of elevator and the overall system performance. In this paper, an LMI-based approach is presented to optimize one single plant parameter, subject to finding a dynamic output-feedback controller that satisfies the closed-loop $H_{\infty}$ performance requirement. Furthermore, polytopic model uncertainties and multi-missions are also considered in the design optimization.

The rest of this paper is organized as follows. In Section II, the integrated plant/output-feedback controller design problem is formulated. Section III presents an LMI-based optimization approach for solving this problem. In section IV, an F-4 fighter aircraft is used to illustrate the design optimization, followed by concluding remarks in Section V. 


\section{Problem Formulation}

Consider a set of polytopic uncertain aircraft models with a varying aircraft parameter as follows.

$$
\mathcal{P}_{i}:\left[\begin{array}{c}
\dot{\mathbf{x}}_{\mathbf{p} i} \\
\mathbf{z}_{i} \\
\mathbf{y}_{\mathbf{p} i}
\end{array}\right]=\left[\begin{array}{ccc}
\mathbf{A}_{\mathbf{p} i}\left(\xi, \Theta_{i}\right) & \mathbf{B}_{\mathbf{p} 1 i}\left(\xi, \Theta_{i}\right) & \mathbf{B}_{\mathbf{p} 2 i}\left(\xi, \Theta_{i}\right) \\
\mathbf{C}_{\mathbf{p} \mathbf{1} i} & \mathbf{0} & \mathbf{D}_{\mathbf{p} \mathbf{2} i} \\
\mathbf{C}_{\mathbf{p} \mathbf{2} i}(\xi) & \mathbf{0} & \mathbf{0}
\end{array}\right]\left[\begin{array}{c}
\mathbf{x}_{\mathbf{p} i} \\
\mathbf{w}_{i} \\
\mathbf{u}_{i}
\end{array}\right]
$$

The subscript $i(i=1,2, \cdots, m)$ represents the linear aircraft model corresponding to the $i$ th mission to be executed. $\mathbf{x}_{\mathbf{p} i}(t) \in \mathbf{R}^{n_{i}}$ is the state vector, $\mathbf{u}_{i}(t) \in$ $\mathbf{R}^{n_{u i}}$ is the control input vector, $\mathbf{y}_{\mathbf{p} i}(t) \in \mathbf{R}^{n_{y i}}$ is the measured output vector, $\mathbf{z}_{i}(t) \in \mathbf{R}^{n_{z i}}$ describes the performance output vector and $\mathbf{w}_{i}(t) \in \mathbf{R}^{n_{w i}}$ is the disturbance vector. For the $i$ th mission, the system matrices $\mathbf{A}_{\mathbf{p} i}\left(\xi, \Theta_{i}\right), \mathbf{B}_{\mathbf{p} \mathbf{1} i}\left(\xi, \Theta_{i}\right), \mathbf{B}_{\mathbf{p} \mathbf{2} i}\left(\xi, \Theta_{i}\right)$ and $\mathbf{C}_{\mathbf{p} 2 i}(\xi)$ are given by

$$
\begin{aligned}
\mathbf{A}_{\mathbf{p} i}\left(\xi, \Theta_{i}\right) & =\sum_{j=1}^{L_{i}}\left(\mathbf{A}_{\mathbf{p} 0 i j}+\xi \mathbf{A}_{\mathbf{p} 1 i j}\right) \theta_{i j} \\
\mathbf{B}_{\mathbf{p} \mathbf{1} i}\left(\xi, \Theta_{i}\right) & =\sum_{j=1}^{L_{i}}\left(\mathbf{B}_{\mathbf{p} \mathbf{1} 0 i j}+\xi \mathbf{B}_{\mathbf{p} \mathbf{1} 1 j}\right) \theta_{i j} \\
\mathbf{B}_{\mathbf{p} \mathbf{2} i}\left(\xi, \Theta_{i}\right) & =\sum_{j=1}^{L_{i}}\left(\mathbf{B}_{\mathbf{p} \mathbf{2} 0 i j}+\xi \mathbf{B}_{\mathbf{p} \mathbf{2} 1 i j}\right) \theta_{i j} \\
\mathbf{C}_{\mathbf{p} \mathbf{2} i}(\xi) & =\mathbf{C}_{\mathbf{p} \mathbf{2} 0 i}+\xi \mathbf{C}_{\mathbf{p} \mathbf{2} 1 i}
\end{aligned}
$$

where the matrices $\mathbf{A}_{\mathbf{p} l i j}, \mathbf{B}_{\mathbf{p} \mathbf{1} l i j}, \mathbf{B}_{\mathbf{p} \mathbf{2} l i j}, \mathbf{C}_{\mathbf{p} \mathbf{2} l i}, \mathbf{C}_{\mathbf{p} \mathbf{1} i}$ and $\mathbf{D}_{\mathbf{p} 12 i}\left(l=0,1, i=1,2, \cdots, m, j=1,2, \cdots, L_{i}\right)$ are known constant matrices of appropriate dimensions, and the parameter $\Theta_{i}=\left[\begin{array}{llll}\theta_{i 1} & \theta_{i 2} & \cdots & \theta_{i L_{i}}\end{array}\right]^{T} \in \mathbf{R}^{L_{i}}$ is the uncertain constant parameter vector and satisfies

$$
\begin{array}{r}
\Theta_{i} \in \Theta \triangleq\left\{\begin{array}{llll}
\theta_{1} & \theta_{2} & \cdots & \theta_{L}
\end{array}\right]^{T} \in \mathbf{R}^{L}: \theta_{j} \geq 0, \\
\left.j=1,2, \cdots, L, \quad \sum_{j=1}^{L} \theta_{j}=1\right\}
\end{array}
$$

Then, for the $i$ th mission, the uncertainty polytope of the system (1) is given by

$\mathcal{O}_{i} \in\left\{\left[\begin{array}{ccc}\mathbf{A}_{\mathbf{p} i}\left(\xi, \Theta_{i}\right) & \mathbf{B}_{\mathbf{p} \mathbf{1} i}\left(\xi, \Theta_{i}\right) & \mathbf{B}_{\mathbf{p} \mathbf{2} i}\left(\xi, \Theta_{i}\right) \\ \mathbf{C}_{\mathbf{p} \mathbf{1} i} & \mathbf{0} & \mathbf{D}_{\mathbf{p} \mathbf{2} i} \\ \mathbf{C}_{\mathbf{p} \mathbf{2} i}(\xi) & \mathbf{0} & \mathbf{0}\end{array}\right], \Theta_{i} \in \boldsymbol{\Theta}\right\}$

which has the following $L_{i}$ vertices

$$
\left[\begin{array}{ccc}
\mathbf{A}_{\mathbf{p} 0 i j}+\xi \mathbf{A}_{\mathbf{p} 1 i j} & \mathbf{B}_{\mathbf{p} 10 i j}+\xi \mathbf{B}_{\mathbf{p} 11 i j} & \mathbf{B}_{\mathbf{p} 20 i j}+\xi \mathbf{B}_{\mathbf{p} 21 i j} \\
\mathbf{C}_{\mathbf{p} 1 i} & \mathbf{0} & \mathbf{D}_{\mathbf{p} 12 i} \\
\mathbf{C}_{\mathbf{p 2 0 i}}+\xi \mathbf{C}_{\mathbf{p} 21 i} & \mathbf{0} & \mathbf{0}
\end{array}\right]
$$

The scalar $\xi$ is the aircraft design parameter to be optimized and belongs to the set

$$
\Xi \triangleq\{\xi \in \mathbf{R}: 0 \leq \xi \leq 1\}
$$

Assume a nominal control surface (e.g., elevator) of size $\bar{S}_{e}$ has been chosen in a prior design stage. The present problem is to determine if this size can be further adjusted downward, i.e., to find a $S_{e} \in\left[\underline{S}_{e} \bar{S}_{e}\right]$ given by $S_{e}=\bar{S}_{e}+\xi\left(\underline{S}_{e}-\bar{S}_{e}\right)$. Hence $\xi=0$ corresponds to the nominal (i.e., largest) and $\xi=1$ corresponds to the smallest allowable size of the control surface.

Assume that the aircraft model with the nominal (i.e., largest) control surface size satisfies

(A1) $\left(\mathbf{A}_{\mathbf{p} 0 i j}, \mathbf{B}_{\mathbf{p} 20 i j}\right)\left(i=1,2, \cdots, m, j=1,2, \cdots, L_{i}\right)$ are stabilizable, respectively;

(A2) $\left(\mathbf{A}_{\mathbf{p} 0 i j}, \mathbf{C}_{\mathbf{p 2 0 i}}\right)\left(i=1,2, \cdots, m, j=1,2, \cdots, L_{i}\right)$ are detectable, respectively.

Consider the following stabilizing dynamic outputfeedback controllers

$$
\mathcal{K}_{i}:\left\{\begin{array}{rl}
\dot{\mathbf{x}}_{\mathbf{k} i} & =\mathbf{A}_{\mathbf{k} i} \mathbf{x}_{\mathbf{k} i}+\mathbf{B}_{\mathbf{k} i} \mathbf{y}_{\mathbf{p} i} \\
\mathbf{u}_{i} & =\mathbf{C}_{\mathbf{k} i} \mathbf{x}_{\mathbf{k} i}+\mathbf{D}_{\mathbf{k} i} \mathbf{y}_{\mathbf{p} i}
\end{array}, i=1,2, \cdots, m\right.
$$

where $\mathbf{x}_{\mathbf{k} i} \in \mathbf{R}^{n_{k i}}$ is the state vector of the dynamic output feedback controller corresponding to the $i$ th mission. Denote this controller by

$$
\mathbf{K}_{i}=\left[\begin{array}{ll}
\mathbf{D}_{\mathbf{k} i} & \mathbf{C}_{\mathbf{k} i} \\
\mathbf{B}_{\mathbf{k} i} & \mathbf{A}_{\mathbf{k} i}
\end{array}\right]
$$

Furthermore, denote

$$
\begin{aligned}
& \mathcal{A}_{i}\left(\xi, \Theta_{i}\right)=\left[\begin{array}{cc}
\mathbf{A}_{\mathbf{p} i}\left(\xi, \Theta_{i}\right) & \mathbf{0} \\
\mathbf{0} & \mathbf{0}
\end{array}\right], \quad \mathbf{x}_{\mathbf{c l} i}=\left[\begin{array}{c}
\mathbf{x}_{\mathbf{p} i} \\
\mathbf{x}_{\mathbf{k} i}
\end{array}\right] \\
& \mathcal{B}_{\mathbf{1} i}\left(\xi, \Theta_{i}\right)=\left[\begin{array}{c}
\mathbf{B}_{\mathbf{p} 1 i}\left(\xi, \Theta_{i}\right) \\
\mathbf{0}
\end{array}\right], \mathcal{C}_{\mathbf{2} i}(\xi)=\left[\begin{array}{cc}
\mathbf{C}_{\mathbf{p} 2 i}(\xi) & \mathbf{0} \\
\mathbf{0} & \mathbf{I}
\end{array}\right] \\
& \mathcal{B}_{\mathbf{2} i}\left(\xi, \Theta_{i}\right)=\left[\begin{array}{cc}
\mathbf{B}_{\mathbf{p} \mathbf{2} i}\left(\xi, \Theta_{i}\right) & \mathbf{0} \\
\mathbf{0} & \mathbf{I}
\end{array}\right] \\
& \mathcal{C}_{\mathbf{1} i}=\left[\begin{array}{c}
\mathbf{C}_{\mathbf{p} 1 i}^{T} \\
\mathbf{0}
\end{array}\right]^{T}, \quad \mathcal{D}_{\mathbf{1 2 i}}=\left[\begin{array}{c}
\mathbf{D}_{\mathbf{p} 12 i}^{T} \\
\mathbf{0}
\end{array}\right]^{T} .
\end{aligned}
$$

Then, the closed-loop systems are described by $\boldsymbol{\Pi}_{i}$

$$
\left[\begin{array}{c}
\dot{\mathbf{x}}_{\mathbf{c l} i} \\
\mathbf{z}_{i}
\end{array}\right]=\left[\begin{array}{cc}
\mathcal{A}_{i}\left(\xi, \Theta_{i}\right)+\mathcal{B}_{\mathbf{2} i}\left(\xi, \Theta_{i}\right) \mathbf{K}_{i} \mathcal{C}_{\mathbf{2}}(\xi) & \mathcal{B}_{\mathbf{1 i}}\left(\xi, \Theta_{i}\right) \\
\mathcal{C}_{\mathbf{1} i}+\mathcal{D}_{\mathbf{1 2} i} \mathbf{K}_{i} \mathcal{C}_{\mathbf{2} i}(\xi) & \mathbf{0}
\end{array}\right]\left[\begin{array}{c}
\mathbf{x}_{\mathbf{c l} i} \\
\mathbf{w}_{i}
\end{array}\right]
$$

Figure 1 gives the block diagram of the closed-loop system (9).

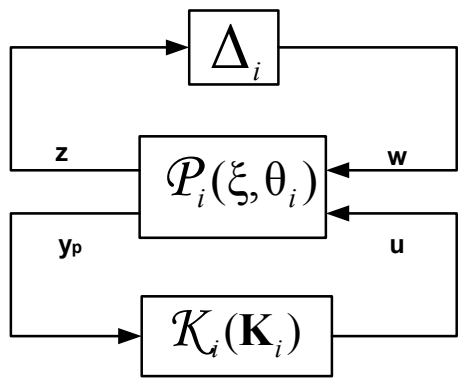

Fig. 1. Interconnection of Plant and Controller

The design objective is to minimize the size of the control surface (that is, to maximize the parameter $\xi$ ) subject to the existence of a set of stabilizing dynamic output-feedback controllers as in (7) satisfying the 
closed-loop performance requirement. In other words, we seek to solve the following optimization problem

$$
\begin{gathered}
\text { maximize } \xi \text { subject to } \\
\left\|\boldsymbol{\Pi}_{\mathbf{z}_{i} \mathbf{w}_{i}}(s)\right\|_{\infty}<\gamma_{i}, \mathbf{K}_{i} \in \mathcal{K}_{i}, \xi \in \Xi, i=1,2, \cdots, m
\end{gathered}
$$

where $\boldsymbol{\Pi}_{\mathbf{z}_{i} \mathbf{w}_{i}}(s)$ denotes the transfer function of the $i$ th closed-loop system of (9) with

$$
\begin{array}{r}
\boldsymbol{\Pi}_{\mathbf{z}_{i} \mathbf{w}_{i}}(s)=\left[\mathcal{C}_{\mathbf{1} i}+\mathcal{D}_{\mathbf{1 2} i} \mathbf{K}_{i} \mathcal{C}_{\mathbf{2} i}(\xi)\right]\left\{s \mathbf{I}-\left[\mathcal{A}_{i}\left(\xi, \Theta_{i}\right)\right.\right. \\
\left.\left.+\mathcal{B}_{\mathbf{2} i}\left(\xi, \Theta_{i}\right) \mathbf{K}_{i} \mathcal{C}_{\mathbf{2} i}(\xi)\right]\right\}^{-1} \mathcal{B}_{\mathbf{1 i}}\left(\xi, \Theta_{i}\right)
\end{array}
$$

Hence, both the aircraft parameter $\xi$ and controller parameters $\mathbf{K}_{i}(i=1,2, \cdots, m)$ are taken into account in the optimization problem (10). Moreover, in the optimization problem (10), aircraft model uncertainties are considered in the form of polytopic uncertainties and multiple missions are also considered simultaneously.

\section{Integrated $H_{\infty}$ Plant/Controller Design}

Lemma 1: [12] Consider the parameter-dependent linear systems $\mathcal{P}_{i}, i=1, \cdots, m$, as in (1) and dynamic output feedback controllers $\mathcal{K}_{i}, i=1, \cdots, m$, of the form (7). For given scalars $\gamma_{i}>0(i=1,2, \cdots, m)$, we have that $\left\|\boldsymbol{\Pi}_{\mathbf{z}_{i} \mathbf{w}_{i}}(s)\right\|_{\infty}<\gamma_{i}(i=1,2, \cdots, m)$ if and only if there exist symmetric positive-definite matrices $\mathbf{Y}_{i} \in \mathbf{R}^{\left(n_{i}+n_{k i}\right) \times\left(n_{i}+n_{k i}\right)} \quad(i=1,2, \cdots, m)$, controller parameter matrices $\mathbf{K}_{i}(i=1,2, \cdots, m)$ as in (8), and a plant parameter $\xi \in \Xi$ such that for all $i=1,2, \cdots, m$,

$$
\left[\begin{array}{cccc}
{\left[\mathcal{A}_{i}\left(\xi, \Theta_{i}\right)+\mathcal{B}_{\mathbf{2} i}\left(\xi, \Theta_{i}\right) \mathbf{K}_{i} \mathcal{C}_{\mathbf{2}}(\xi)\right]^{T} \mathbf{Y}_{i}} & & \\
+\mathbf{Y}_{i}\left[\mathcal{A}_{i}\left(\xi, \Theta_{i}\right)+\mathcal{B}_{\mathbf{2} i}\left(\xi, \Theta_{i}\right) \mathbf{K}_{i} \mathcal{C}_{\mathbf{2}}(\xi)\right] & * & * \\
\mathcal{B}_{\mathbf{1} i}^{T}\left(\xi, \Theta_{i}\right) \mathbf{Y}_{i} & -\gamma_{i} \mathbf{I} & * \\
\mathcal{C}_{\mathbf{1} i}+\mathcal{D}_{\mathbf{1 2} i} \mathbf{K}_{i} \mathcal{C}_{\mathbf{2} i}(\xi) & \mathbf{0} & -\gamma_{i} \mathbf{I}
\end{array}\right]<0
$$

Note that $*$ denotes symmetric entries of a symmetric matrix. It is applicable to the rest of this paper.

Remark 1: Lemma 1 gives a necessary and sufficient condition to solve the integrated plant/ output-feedback controller design problem. However, as $\xi, \mathbf{Y}_{i}$ and $\mathbf{K}_{i}$ $(i=1,2, \cdots, m)$ are variables, the condition (12) is not an LMI. To be numerically solvable, it needs to be transformed into an LMI.

In the following, an important lemma is introduced.

Lemma 2: (Projection Lemma)[1] Given a symmetric matrix $\boldsymbol{\Psi}$ and two matrices $\mathbf{P}$ and $\mathbf{Q}$, there exists an $\mathbf{X}$ such that the following LMI holds

$$
\mathbf{\Psi}+\mathbf{P}^{T} \mathbf{X}^{T} \mathbf{Q}+\mathbf{Q}^{T} \mathbf{X P}<0
$$

if and only if the following projection inequalities are satisfied

$$
\mathcal{N}_{P}^{T} \boldsymbol{\Psi} \mathcal{N}_{P}<0, \quad \mathcal{N}_{Q}^{T} \boldsymbol{\Psi} \mathcal{N}_{Q}<0
$$

where $\mathcal{N}_{P}$ and $\mathcal{N}_{Q}$ denote arbitrary bases of the null spaces of $\mathbf{P}$ and $\mathbf{Q}$, respectively.

Since the inequality (12) can be rewritten as

$$
\left[\begin{array}{ccc}
\mathcal{A}_{i}^{T}\left(\xi, \Theta_{i}\right) \mathbf{Y}_{i}+\mathbf{Y}_{i} \mathcal{A}_{i}\left(\xi, \Theta_{i}\right) & \mathbf{Y}_{i} \mathcal{B}_{\mathbf{1} i}\left(\xi, \Theta_{i}\right) & \mathcal{C}_{\mathbf{1} i}^{T} \\
\mathcal{B}_{\mathbf{1} i}^{T}\left(\xi, \Theta_{i}\right) \mathbf{Y}_{i} & -\gamma_{i} \mathbf{I} & \mathbf{0} \\
\mathcal{C}_{\mathbf{1} i} & \mathbf{0} & -\gamma_{i} \mathbf{I}
\end{array}\right]+
$$

$$
\left[\begin{array}{c}
\mathbf{Y}_{i} \mathcal{B}_{\mathbf{2} i}\left(\xi, \Theta_{i}\right) \\
\mathbf{0} \\
\mathcal{D}_{\mathbf{1 2} i}
\end{array}\right] \mathbf{K}_{i}\left[\begin{array}{c}
\mathcal{C}_{\mathbf{2} i}^{T}(\xi) \\
\mathbf{0} \\
\mathbf{0}
\end{array}\right]^{T}+\left[\begin{array}{c}
\mathcal{C}_{\mathbf{2} i}^{T}(\xi) \\
\mathbf{0} \\
\mathbf{0}
\end{array}\right] \mathbf{K}_{i}^{T}\left[\begin{array}{c}
\mathbf{Y}_{i} \mathcal{B}_{\mathbf{2 i}}\left(\xi, \Theta_{i}\right) \\
\mathbf{0} \\
\mathcal{D}_{\mathbf{1 2} i}
\end{array}\right]^{T}<0
$$

according to Lemma 2 , it is equivalent to

$$
\begin{gathered}
\mathcal{N}_{P}^{T}\left[\begin{array}{ccc}
\mathcal{A}_{i}^{T}\left(\xi, \Theta_{i}\right) \mathbf{Y}_{i}+\mathbf{Y}_{i} \mathcal{A}_{i}\left(\xi, \Theta_{i}\right) & * & * \\
\mathcal{B}_{\mathbf{1} i}^{T}\left(\xi, \Theta_{i}\right) \mathbf{Y}_{i} & -\gamma_{\mathbf{i}} \mathbf{I} & * \\
\mathcal{C}_{\mathbf{1}} & \mathbf{0} & -\gamma_{i} \mathbf{I}
\end{array}\right] \mathcal{N}_{P}<0 \\
\mathcal{N}_{Q}^{T}\left[\begin{array}{ccc}
\mathcal{A}_{i}^{T}\left(\xi, \Theta_{i}\right) \mathbf{Y}_{i}+\mathbf{Y}_{i} \mathcal{A}_{i}\left(\xi, \Theta_{i}\right) & * & * \\
\mathcal{B}_{\mathbf{1}}^{T}\left(\xi, \Theta_{i}\right) \mathbf{Y}_{i} & -\gamma_{i} \mathbf{I} & * \\
\mathcal{C}_{\mathbf{1}} & \mathbf{0} & -\gamma_{i} \mathbf{I}
\end{array}\right] \mathcal{N}_{Q}<0
\end{gathered}
$$

where $\mathcal{N}_{P}$ and $\mathcal{N}_{Q}$ are the bases of the null spaces of

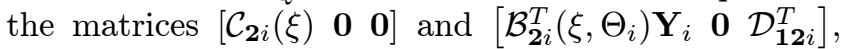
respectively. It is noted that the controller parameter $\mathbf{K}_{i}$ is no longer included in the inequalities (16) and (17). Hence, the integrated plant/controller optimization design problem can be separated into the optimization design problem of plant parameter $\xi$ and that of controller parameters $\mathbf{K}_{i}(i=1,2, \cdots, m)$. In the following, the plant parameter and controller parameters are individually optimized.

\section{A. Design Optimization of Plant Parameter}

Partition $\mathbf{Y}_{i}$ and $\mathbf{Y}_{i}^{-1}$ as

$$
\mathbf{Y}_{i}=\left[\begin{array}{cc}
\mathbf{S}_{i} & \mathbf{N}_{i} \\
\mathbf{N}_{i}^{T} & \#
\end{array}\right], \quad \mathbf{Y}_{i}^{-1}=\left[\begin{array}{cc}
\mathbf{R}_{i} & \mathbf{M}_{i} \\
\mathbf{M}_{i}^{T} & \$
\end{array}\right]
$$

and denote

$$
\mathbf{Z}_{\mathbf{1} i}=\left[\begin{array}{cc}
\mathbf{I} & \mathbf{R}_{i} \\
\mathbf{0} & \mathbf{M}_{i}^{T}
\end{array}\right], \quad \mathbf{Z}_{\mathbf{2} i}=\left[\begin{array}{cc}
\mathbf{S}_{i} & \mathbf{I} \\
\mathbf{N}_{i}^{T} & \mathbf{0}
\end{array}\right]
$$

It is easy to see that

$$
\mathbf{R}_{i} \mathbf{S}_{i}+\mathbf{M}_{i} \mathbf{N}_{i}^{T}=\mathbf{I}, \quad \mathbf{Y}_{i} \mathbf{Z}_{\mathbf{1} i}=\mathbf{Z}_{\mathbf{2} i}
$$

Multiplying $\mathbf{Z}_{\mathbf{1} i}^{T}$ and $\mathbf{Z}_{\mathbf{1} i}$ on the left and right sides of $\mathbf{Y}_{i}>0$, respectively, we obtain

$$
\left[\begin{array}{cc}
\mathbf{R}_{i} & \mathbf{I} \\
\mathbf{I} & \mathbf{S}_{i}
\end{array}\right] \geq 0
$$

\section{Denote}

$$
\begin{aligned}
& \Delta_{\mathbf{1} i j}\left(\mathbf{S}_{i}, \mathbf{E}_{i}, \gamma_{i}\right)= \\
& {\left[\begin{array}{ccc}
\mathbf{A}_{\mathbf{p} 0 i j}^{T} \mathbf{S}_{i}+\mathbf{S}_{i} \mathbf{A}_{\mathbf{p} 0 i j} & \mathbf{S}_{i} \mathbf{B}_{\mathbf{p} 10 i j} & \mathbf{C}_{\mathbf{p} 1 i}^{T} \\
\mathbf{B}_{\mathbf{p} 10 i j}^{T} \mathbf{S}_{i} & -\gamma_{i} \mathbf{I} & \mathbf{0} \\
\mathbf{C}_{\mathbf{p} 1 i} & \mathbf{0} & -\gamma_{i} \mathbf{I}
\end{array}\right]} \\
& +\mathbf{E}_{i}\left[\begin{array}{c}
\mathbf{C}_{\mathbf{p 2 0 i}}^{T} \\
\mathbf{0} \\
\mathbf{0}
\end{array}\right]^{T}+\left[\begin{array}{c}
\mathbf{C}_{\mathbf{p 2 0 i}}^{T} \\
\mathbf{0} \\
\mathbf{0}
\end{array}\right] \mathbf{E}_{i}^{T} \\
& \Delta_{\mathbf{2} i j}\left(\mathbf{S}_{i}, \mathbf{E}_{i}\right)= \\
& {\left[\begin{array}{ccc}
\mathbf{A}_{\mathbf{p} 1 i j}^{T} \mathbf{S}_{i}+\mathbf{S}_{i} \mathbf{A}_{\mathbf{p} 1 i j} & \mathbf{S}_{i} \mathbf{B}_{\mathbf{p} 11 i j} & \mathbf{0} \\
\mathbf{B}_{\mathbf{p} 11 i j}^{T} \mathbf{S}_{i} & \mathbf{0} & \mathbf{0} \\
\mathbf{0} & \mathbf{0} & \mathbf{0}
\end{array}\right]} \\
& +\mathbf{E}_{i}\left[\begin{array}{c}
\mathbf{C}_{\mathbf{p 2 1 i}}^{T} \\
\mathbf{0} \\
\mathbf{0}
\end{array}\right]^{T}+\left[\begin{array}{c}
\mathbf{C}_{\mathbf{p 2 1 i}}^{T} \\
\mathbf{0} \\
\mathbf{0}
\end{array}\right] \mathbf{E}_{i}^{T}
\end{aligned}
$$




$$
\begin{aligned}
& \Delta_{\mathbf{3} i j}\left(\mathbf{R}_{i}, \mathbf{H}_{i}, \gamma_{i}\right)= \\
& {\left[\begin{array}{ccc}
\mathbf{A}_{\mathbf{p} 0 i j} \mathbf{R}_{i}+\mathbf{R}_{i} \mathbf{A}_{\mathbf{p} 0 i j}^{T} & \mathbf{B}_{\mathbf{p} 10 i j} & \mathbf{R}_{i} \mathbf{C}_{\mathbf{p} 1 i}^{T} \\
\mathbf{B}_{\mathbf{p} 10 i j}^{T} & -\gamma_{i} \mathbf{I} & \mathbf{0} \\
\mathbf{C}_{\mathbf{p} 1 i} \mathbf{R}_{i} & \mathbf{0} & -\gamma_{i} \mathbf{I}
\end{array}\right]} \\
& +\mathbf{H}_{i}\left[\begin{array}{c}
\mathbf{B}_{\mathbf{p 2 0 i j}} \\
\mathbf{0} \\
\mathbf{D}_{\mathbf{p 1 2 i}}
\end{array}\right]^{T}+\left[\begin{array}{c}
\mathbf{B}_{\mathbf{p 2 0 i j}} \\
\mathbf{0} \\
\mathbf{D}_{\mathbf{p} 12 i}
\end{array}\right] \mathbf{H}_{i}^{T} \\
& \Delta_{\mathbf{4} i j}\left(\mathbf{R}_{i}, \mathbf{H}_{i}\right)= \\
& {\left[\begin{array}{ccc}
\mathbf{A}_{\mathbf{p} 1 i j} \mathbf{R}_{i}+\mathbf{R}_{i} \mathbf{A}_{\mathbf{p} 1 i j}^{T} & \mathbf{B}_{\mathbf{p} 11 i j} & \mathbf{0} \\
\mathbf{B}_{\mathbf{p} 11 i j}^{T} & \mathbf{0} & \mathbf{0} \\
\mathbf{0} & \mathbf{0} & \mathbf{0}
\end{array}\right]} \\
& +\mathbf{H}_{i}\left[\begin{array}{c}
\mathbf{B}_{\mathbf{p 2 1 i j}} \\
\mathbf{0} \\
\mathbf{0}
\end{array}\right]^{T}+\left[\begin{array}{c}
\mathbf{B}_{\mathbf{p 2 1 i j}} \\
\mathbf{0} \\
\mathbf{0}
\end{array}\right] \mathbf{H}_{i}^{T}
\end{aligned}
$$

with $\mathbf{E}_{i} \in \mathbf{R}^{\left(n_{i}+n_{w i}+n_{z i}\right) \times n_{y i}}$ and $\mathbf{H}_{i} \in \mathbf{R}^{\left(n_{i}+n_{w i}+n_{z i}\right) \times n_{u i}}$.

Theorem 1: For given scalar $\gamma_{i}>0(i=1,2, \cdots, m)$, if there exist a plant parameter $\xi \in \Xi$, symmetric positive-definite matrices $\mathbf{S}_{i} \in \mathbf{R}^{n_{i} \times n_{i}}$ and $\mathbf{R}_{i} \in$ $\mathbf{R}^{n_{i} \times n_{i}}$, and matrices $\mathbf{E}_{i}$ and $\mathbf{H}_{i}(i=1,2, \cdots, m)$ such that for all $i=1,2, \cdots, m$ and $j=1,2, \cdots, L_{i}$,

$$
\begin{aligned}
\Delta_{\mathbf{1} i j}\left(\mathbf{S}_{i}, \mathbf{E}_{i}, \gamma_{i}\right)+\xi \Delta_{\mathbf{2} i j}\left(\mathbf{S}_{i}, \mathbf{E}_{i}\right) & <0 \\
\Delta_{\mathbf{3} i j}\left(\mathbf{R}_{i}, \mathbf{H}_{i}, \gamma_{i}\right)+\xi \Delta_{\mathbf{4} i j}\left(\mathbf{R}_{i}, \mathbf{H}_{i}\right) & <0 \\
{\left[\begin{array}{cc}
\mathbf{R}_{i} & \mathbf{I} \\
\mathbf{I} & \mathbf{S}_{i}
\end{array}\right] } & \geq 0
\end{aligned}
$$

then there exist dynamic output-feedback control matrices $\mathbf{K}_{i}(i=1,2, \cdots, m)$ as in (8) such that the closed-loop system $\boldsymbol{\Pi}_{i}(i=1,2, \cdots, m)$ in (9) are robustly stabilized and satisfy $\left\|\boldsymbol{\Pi}_{\mathbf{z}_{i} \mathbf{w}_{i}}(s)\right\|_{\infty}<\gamma_{i}(i=$ $1,2, \cdots, m)$.

Proof: Due to the space limitation, the proof is omitted.

Now the optimization problem of the plant parameter $\xi$ is equivalent to a generalized eigenvalue problem (GEVP) stated as follows: minimize $\lambda$ subject to the following LMIs

$$
\begin{aligned}
& \Delta_{\mathbf{1} i j}\left(\mathbf{S}_{i}, \mathbf{E}_{i}, \gamma_{i}\right)<0 \\
& \Delta_{\mathbf{3} i j}\left(\mathbf{R}_{i}, \mathbf{H}_{i}, \gamma_{i}\right)<0 \\
& \gamma_{i}<\gamma_{i 0} \\
& {\left[\begin{array}{cc}
\mathbf{R}_{i} & \mathbf{I} \\
\mathbf{I} & \mathbf{S}_{i}
\end{array}\right] } \geq 0 \\
& \Delta_{\mathbf{2} i j}\left(\mathbf{S}_{i}, \mathbf{E}_{i}\right)<-\lambda \Delta_{\mathbf{1} i j}\left(\mathbf{S}_{i}, \mathbf{E}_{i}, \gamma_{i}\right) \\
& \Delta_{\mathbf{4} i j}\left(\mathbf{R}_{i}, H\right)<-\lambda \Delta_{\mathbf{3} i j}\left(\mathbf{R}_{i}, \mathbf{H}_{i}, \gamma_{i}\right) \\
& i=1,2, \cdots, m, \quad j=1,2, \cdots, L_{i}
\end{aligned}
$$

where $\gamma_{i 0}(i=1,2, \cdots, m)$ are given closed-loop $H_{\infty}$ performance upper bounds corresponding to the $i$ th mission. Then the optimized plant parameter $\xi_{\text {opt }}=$ $1 / \lambda_{\text {opt }}$.

Remark 2: Theorem 1 gives a sufficient condition for the optimization of the aircraft parameter $\xi$. Once the optimal $\xi_{\text {opt }}$ is obtained, the optimal dynamic outputfeedback controllers $\mathbf{K}_{i}(i=1,2, \cdots, m)$ as in (8) can be solved by using the following approach.

\section{B. Design Optimization of Controllers}

With the optimized plant parameter $\xi_{\text {opt }}$ from Section IIIA, we now look at the optimization of the controller parameters.

Performing a congruence transformation with $\operatorname{diag}\left\{\mathbf{Z}_{\mathbf{1} i}, \mathbf{I}, \mathbf{I}\right\}$ on the matrix inequality (12), we have the following equivalent matrix inequality

$$
\begin{aligned}
& {\left[\mathbf{Z}_{1 i}^{T}\left[\mathcal{A}_{i}\left(\xi_{\text {opt }}, \Theta_{i}\right)+\mathcal{B}_{\mathbf{2} i}\left(\xi_{\text {opt }}, \Theta_{i}\right) \mathbf{K}_{i} \mathcal{C}_{\mathbf{2} i}\left(\xi_{\text {opt }}\right)\right]^{T} \mathbf{Z}_{\mathbf{2} i}\right.} \\
& +\mathbf{Z}_{\mathbf{2} i}^{T}\left[\mathcal{A}_{i}\left(\xi_{\text {opt }}, \Theta_{i}\right)+\mathcal{B}_{\mathbf{2} i}\left(\xi_{\text {opt }}, \Theta_{i}\right) \mathbf{K}_{i} \mathcal{C}_{\mathbf{2} i}\left(\xi_{\text {opt }}\right)\right] \mathbf{Z}_{\mathbf{1} i} \\
& \mathcal{B}_{\mathbf{1 i}}^{T}\left(\xi_{\text {opt }}, \Theta_{i}\right) \mathbf{Z}_{\mathbf{2} i} \\
& {\left[\mathcal{C}_{\mathbf{1} i}+\mathcal{D}_{\mathbf{1 2 i}} \mathbf{K}_{i} \mathcal{C}_{\mathbf{2} i}\left(\xi_{\text {opt }}\right)\right] \mathbf{Z}_{\mathbf{1} i}} \\
& \left.\begin{array}{cc}
* & * \\
-\gamma_{i} \mathbf{I} & * \\
\mathbf{0} & -\gamma_{i} \mathbf{I}
\end{array}\right]<0(31)
\end{aligned}
$$

Partition $\mathbf{Z}_{\mathbf{1} i}$ and $\mathbf{Z}_{\mathbf{2} i}$ as in (19), then (31) can be rewritten as

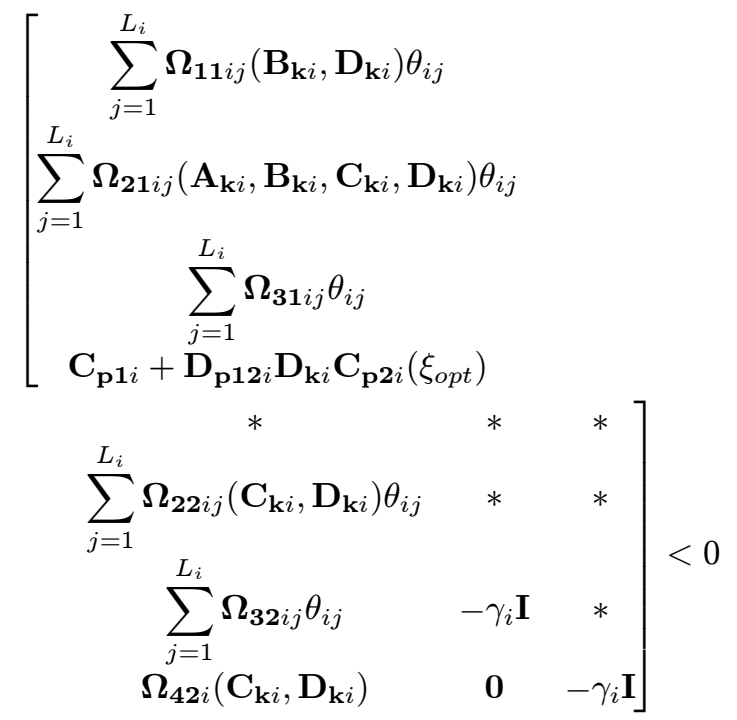

where

$$
\begin{aligned}
& \boldsymbol{\Omega}_{\mathbf{1 1} i j}\left(\mathbf{B}_{\mathbf{k} i}, \mathbf{D}_{\mathbf{k} i}\right)= \\
& \mathbf{S}_{i}\left(\mathbf{A}_{\mathbf{p} 0 i j}+\xi_{\text {opt }} \mathbf{A}_{\mathbf{p} 1 i j}\right)+\left(\mathbf{A}_{\mathbf{p} 0 i j}^{T}+\xi_{\text {opt }} \mathbf{A}_{\mathbf{p} 1 i j}^{T}\right) \mathbf{S}_{i} \\
& +\mathbf{C}_{\mathbf{p} 2 i}^{T}\left(\xi_{\text {opt }}\right)\left[\mathbf{N}_{i} \mathbf{B}_{\mathbf{k} i}+\mathbf{S}_{i}\left(\mathbf{B}_{\mathbf{p} 20 i j}+\xi_{\text {opt }} \mathbf{B}_{\mathbf{p} 21 i j}\right) \mathbf{D}_{\mathbf{k} i}\right]^{T} \\
& +\left[\mathbf{N}_{i} \mathbf{B}_{\mathbf{k} i}+\mathbf{S}_{i}\left(\mathbf{B}_{\mathbf{p} 20 i j}+\xi_{\text {opt }} \mathbf{B}_{\mathbf{p 2 1 i j}}\right) \mathbf{D}_{\mathbf{k} i}\right] \mathbf{C}_{\mathbf{p} 2 i}\left(\xi_{\text {opt }}\right) \\
& \boldsymbol{\Omega}_{\mathbf{2 1} i j}\left(\mathbf{A}_{\mathbf{k} i}, \mathbf{B}_{\mathbf{k} i}, \mathbf{C}_{\mathbf{k} i}, \mathbf{D}_{\mathbf{k} i}\right)= \\
& \mathbf{R}_{i} \mathbf{C}_{\mathbf{p} \mathbf{2} i}^{T}\left(\xi_{\text {opt }}\right) \mathbf{B}_{\mathbf{k} i}^{T} \mathbf{N}_{i}^{T}+\mathbf{M}_{i} \mathbf{C}_{\mathbf{k} i}^{T}\left(\mathbf{B}_{\mathbf{p} \mathbf{2} 0 i j}^{T}+\xi_{\text {opt }} \mathbf{B}_{\mathbf{p} \mathbf{2} 1 i j}^{T}\right) \mathbf{S}_{i} \\
& +\mathbf{M}_{i} \mathbf{A}_{\mathbf{k} i}^{T} \mathbf{N}_{i}^{T}+\left(\mathbf{B}_{\mathbf{p} 20 i j}+\xi_{\text {opt }} \mathbf{B}_{\mathbf{p} 21 i j}\right) \mathbf{D}_{\mathbf{k} i} \mathbf{C}_{\mathbf{p} 2 i}\left(\xi_{\text {opt }}\right) \\
& +\mathbf{R}_{i}\left(\mathbf{A}_{\mathbf{p} 0 i j}^{T}+\xi_{\text {opt }} \mathbf{A}_{\mathbf{p} 1 i j}^{T}\right) \mathbf{S}_{i}+\left(\mathbf{A}_{\mathbf{p} 0 i j}+\xi_{\text {opt }} \mathbf{A}_{\mathbf{p} 1 i j}\right) \\
& +\mathbf{R}_{i} \mathbf{C}_{\mathbf{p} 2 i}^{T}\left(\xi_{\text {opt }}\right) \mathbf{D}_{\mathbf{k} i}^{T}\left(\mathbf{B}_{\mathbf{p} 20 i j}^{T}+\xi_{\text {opt }} \mathbf{B}_{\mathbf{p} 21 i j}^{T}\right) \mathbf{S}_{i} \\
& \boldsymbol{\Omega}_{\mathbf{2 2} i j}\left(\mathbf{C}_{\mathbf{k} i}, \mathbf{D}_{\mathbf{k} i}\right)= \\
& \left(\mathbf{A}_{\mathbf{p} 0 i j}+\xi_{\text {opt }} \mathbf{A}_{\mathbf{p} 1 i j}\right) \mathbf{R}_{i}+\mathbf{R}_{i}\left(\mathbf{A}_{\mathbf{p} 0 i j}^{T}+\xi_{o p t} \mathbf{A}_{\mathbf{p} 1 i j}^{T}\right) \\
& +\left(\mathbf{B}_{\mathbf{p} \mathbf{2} 0 i j}+\xi_{\text {opt }} \mathbf{B}_{\mathbf{p} \mathbf{2} 1 i j}\right)\left[\mathbf{C}_{\mathbf{k} i} \mathbf{M}_{i}^{T}+\mathbf{D}_{\mathbf{k} i} \mathbf{C}_{\mathbf{p} \mathbf{2} i}\left(\xi_{\text {opt }}\right) \mathbf{R}_{i}\right] \\
& +\left[\mathbf{C}_{\mathbf{k} i} \mathbf{M}_{i}^{T}+\mathbf{D}_{\mathbf{k} i} \mathbf{C}_{\mathbf{p} 2 i}\left(\xi_{\text {opt }}\right) \mathbf{R}_{i}\right]^{T}\left(\mathbf{B}_{\mathbf{p} 20 i j}^{T}+\xi_{\text {opt }} \mathbf{B}_{\mathbf{p} 21 i j}^{T}\right)
\end{aligned}
$$




$$
\begin{aligned}
& \boldsymbol{\Omega}_{\mathbf{3 1 i j}}=\left(\mathbf{B}_{\mathbf{p} \mathbf{1} 0 i j}^{T}+\xi_{\text {opt }} \mathbf{B}_{\mathbf{p} \mathbf{1} 1 j}^{T}\right) \mathbf{S}_{i} \\
& \boldsymbol{\Omega}_{\mathbf{3 2 i j}}=\mathbf{B}_{\mathbf{p} \mathbf{1} 0 i j}^{T}+\xi_{\text {opt }} \mathbf{B}_{\mathbf{p} 11 i j}^{T} \\
& \boldsymbol{\Omega}_{\mathbf{4 2 i} i}\left(\mathbf{C}_{\mathbf{k} i}, \mathbf{D}_{\mathbf{k} i}\right)= \\
& \mathbf{C}_{\mathbf{p} \mathbf{1} i} \mathbf{R}_{i}+\mathbf{D}_{\mathbf{p} \mathbf{1} i}\left[\mathbf{C}_{\mathbf{k} i} \mathbf{M}_{i}^{T}+\mathbf{D}_{\mathbf{k} i} \mathbf{C}_{\mathbf{p} \mathbf{2} i}\left(\xi_{\text {opt }}\right) \mathbf{R}_{i}\right]
\end{aligned}
$$

Now the optimal dynamic output-feedback controllers $K_{\text {iopt }}(i=1,2, \cdots, m)$ can be solved as follows:

Step 1 For a given $\xi_{\text {opt }}$, minimize $\gamma_{i}$ subject to (24) and

$$
\begin{array}{r}
\Delta_{\mathbf{1} i j}\left(\mathbf{S}_{i}, \mathbf{E}_{i}, \gamma_{i}\right)+\xi_{\text {opt }} \Delta_{\mathbf{2} i j}\left(\mathbf{S}_{i}, \mathbf{E}_{i}\right)<0 \\
\Delta_{\mathbf{3} i j}\left(\mathbf{R}_{i}, \mathbf{H}_{i}, \gamma_{i}\right)+\xi_{\text {opt }} \Delta_{\mathbf{4} i j}\left(\mathbf{R}_{i}, \mathbf{H}_{i}\right)<0 \\
j=1,2, \cdots, L_{i}
\end{array}
$$

Then we obtain $\mathbf{S}_{\text {iopt }}$ and $\mathbf{R}_{\text {iopt }}(i=1,2, \cdots, m)$. Once invertible matrices $\mathbf{M}_{\text {iopt }}(i=1,2, \cdots, m)$ are chosen, we have $\mathbf{N}_{\text {iopt }}=\left(\mathbf{I}-\mathbf{S}_{\text {iopt }} \mathbf{R}_{\text {iopt }}\right)\left(\mathbf{M}_{\text {iopt }}^{-1}\right)^{T}(i=$ $1,2, \cdots, m)$.

Step 2 Let $\mathbf{S}_{i}=\mathbf{S}_{\text {iopt }}, \mathbf{R}_{i}=\mathbf{R}_{\text {iopt }}, \mathbf{M}_{i}=\mathbf{M}_{\text {iopt }}$ and $\mathbf{N}_{i}=\mathbf{N}_{\text {iopt }}$ where $\mathbf{S}_{\text {iopt }}, \mathbf{R}_{\text {iopt }}, \mathbf{M}_{\text {iopt }}$ and $\mathbf{N}_{\text {iopt }}$ are obtained from Step 1. Minimize $\gamma_{i}$ subject to

$$
\begin{aligned}
& {\left[\begin{array}{cc}
\boldsymbol{\Omega}_{\mathbf{1 1} i j}\left(\mathbf{B}_{\mathbf{k} i}, \mathbf{D}_{\mathbf{k} i}\right) & * \\
\boldsymbol{\Omega}_{\mathbf{2 1} i j}\left(\mathbf{A}_{\mathbf{k} i}, \mathbf{B}_{\mathbf{k} i}, \mathbf{C}_{\mathbf{k} i}, \mathbf{D}_{\mathbf{k} i}\right) & \boldsymbol{\Omega}_{\mathbf{2 2} i j}\left(\mathbf{C}_{\mathbf{k} i}, \mathbf{D}_{\mathbf{k} i}\right) \\
\boldsymbol{\Omega}_{\mathbf{3 1 i j}} & \boldsymbol{\Omega}_{\mathbf{3 2 i j}} \\
\mathbf{C}_{\mathbf{p} \mathbf{1} i}+\mathbf{D}_{\mathbf{p} \mathbf{1 2} i} \mathbf{D}_{\mathbf{k} i} \mathbf{C}_{\mathbf{p} \mathbf{2} i}\left(\xi_{\text {opt }}\right) & \boldsymbol{\Omega}_{\mathbf{4 2 i}}\left(\mathbf{C}_{\mathbf{k} i}, \mathbf{D}_{\mathbf{k} i}\right)
\end{array}\right.} \\
& \left.\begin{array}{cc}
* & * \\
* & * \\
-\gamma_{i} \mathbf{I} & * \\
\mathbf{0} & -\gamma_{i} \mathbf{I}
\end{array}\right]<0
\end{aligned}
$$

Then we have $\mathbf{A}_{\mathbf{k} i o p t}, \mathbf{B}_{\mathbf{k} i o p t}, \mathbf{C}_{\mathbf{k} i o p t}, \mathbf{D}_{\mathbf{k} i o p t}$.

Remark 3: For integrated plant/controller design optimization, the optimization of plant parameter can be separated from the optimization of controller parameters by using the projection lemma. For the singleparameter case, the corresponding plant parameter optimization problem can be reformulated as a GEVP problem. However, this is not true for the multi-plantparameter case. The integrated plant/controller optimization design problem for the multi-plant-parameter case is addressed in the paper [5].

\section{Example}

In this section, a polytopic uncertain model of the longitudinal dynamics of an F-4 fighter aircraft [2] is used to demonstrate the proposed integrated plant/controller optimization approach. A mission with the following system performance requirements is considered.

- The closed-loop system must be internally stable.

- There should be no steady-state error in the response to step commands in the angle of attack $\alpha$ and flight path angle $\gamma(=\theta-\alpha, \theta$ is pitch angle).

- A flight path angle of 1 degree must be generated using elevator and spoiler deflection of less than 20 and 40 degrees, respectively.
The design objective is to minimize the size of the elevator of the F-4 aircraft (i.e., maximize $\xi, \xi \in[0,1]$ ) subject to the existence of a dynamic output-feedback controller that satisfies the above system performance requirements.

To achieve zero steady-state tracking errors of the angle of attack and flight path angle, the augumented aircraft system is described by (1) with $m=1$,

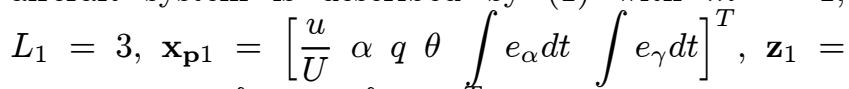
$\left[\begin{array}{llllll}\delta_{e} & \delta_{s} & \delta_{t} & \alpha & \int e_{\alpha} d t & \int e_{\gamma} d t\end{array}\right]^{T}, \mathbf{w}_{1}=\left[\begin{array}{ll}\mathbf{w}_{g 1} & \gamma_{c}\end{array}\right]^{T}$, and

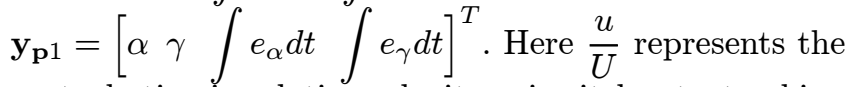
perturbation in relative velocity; $q$ is pitch rate; tracking error $e_{\alpha}=\alpha_{c}-\alpha$ with $\alpha_{c}$ being command signal of angle of attack $\alpha$; tracking error $e_{\gamma}=\gamma_{c}-\gamma$ with $\gamma_{c}$ being command signal of flight path angle $\gamma ; \mathbf{w}_{g 1}$ represents the vertical gust disturbance; $\delta_{e}$ represents elevator deflection; $\delta_{s}$ represents spoiler deflection; and $\delta_{t}$ represents thrust.

Applying the GEVP method for plant parameter optimization proposed in Section IIIA, we obtain the optimal design parameter $\xi_{\text {opt }}=0.7432$ when the design performance index $\gamma_{10}=1.1$ is chosen. Here, the larger $\xi_{\text {opt }}$ is, the smaller the size of elevator. In this example, $\xi_{\text {opt }}=0.7432$ indicates that the fighter aircraft can be stabilized and perform tracking task with a much smaller elevator size. Applying the optimization approach for controller parameters proposed in Section IIIB, we obtain an optimal dynamic output-feedback controller that achieves the optimal closed-loop $H_{\infty}$ performance $\gamma_{1 o p t}=1.09$. It is noted that there is a very small gap between the designed $H_{\infty}$ performance upper bound $\gamma_{10}=1.1$ and the achieved $H_{\infty}$ performance upper bound $\gamma_{1 o p t}=1.09$. It indicates that solution for optimal plant parameter has almost no conservativeness although only a sufficient condition is provided for optimization of plant parameter. This can be further verified by Figure 2, which shows the curve of the closedloop $H_{\infty}$ performance vs the plant parameter $\xi$. Here, for each $\xi$ and $\Theta_{1}$ obtained by using grid method, the closed-loop $H_{\infty}$ performance is calculated by using the approach proposed by Scherer, et al [9].

To compare the performance of the aircraft with $\xi=0.7432$ to the performance of the nominal aircraft with $\xi=0$, Figure 3 gives the simulation results of the aircraft with $\xi=0$ (see the red lines) and $\xi=0.7432$ (see the black lines), respectively, where the solid lines represent the responses with the uncertain parameter vector $\Theta_{1}=\left[\begin{array}{lll}1 & 0 & 0\end{array}\right]$, the dash lines represent the responses with $\Theta_{1}=\left[\begin{array}{lll}0 & 1 & 0\end{array}\right]$, and the dash-dotted lines represent the responses with $\Theta_{1}=\left[\begin{array}{lll}0 & 0 & 1\end{array}\right]$. Compared to the responses with $\xi=0$, there is no obvious difference in the responses of the path angle $\gamma$, the angle of attack $\alpha$ and the spoiler deflection with $\xi=0.7432$. 


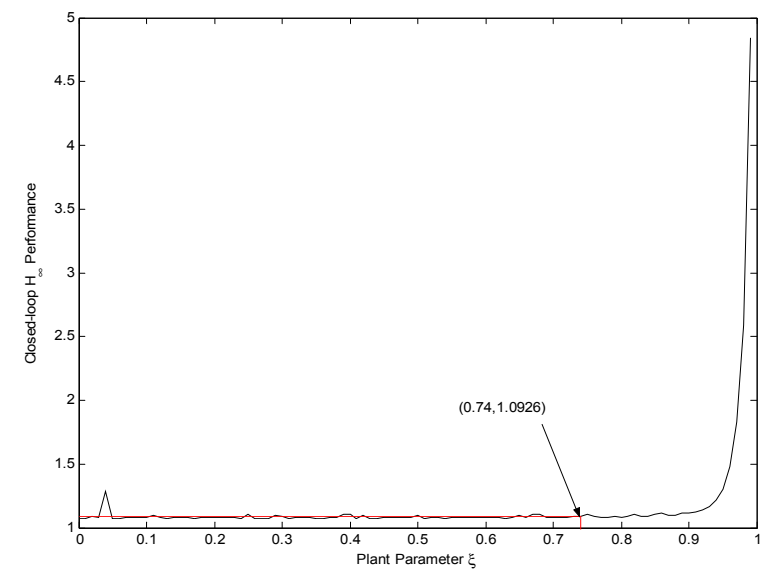

Fig. 2. Curve of closed-loop $H_{\infty}$ performance vs plant parameter $\xi$

However, the elevator deflections with $\xi=0.7432$ are larger than those with $\xi=0$. It should be stressed that usually the size of elevator cannot be as small as in this example because only one mission is considered here. In practice, multiple missions and large uncertainties as well as more realistic disturbances need be considered simultaneously, resulting in much larger elevator size.
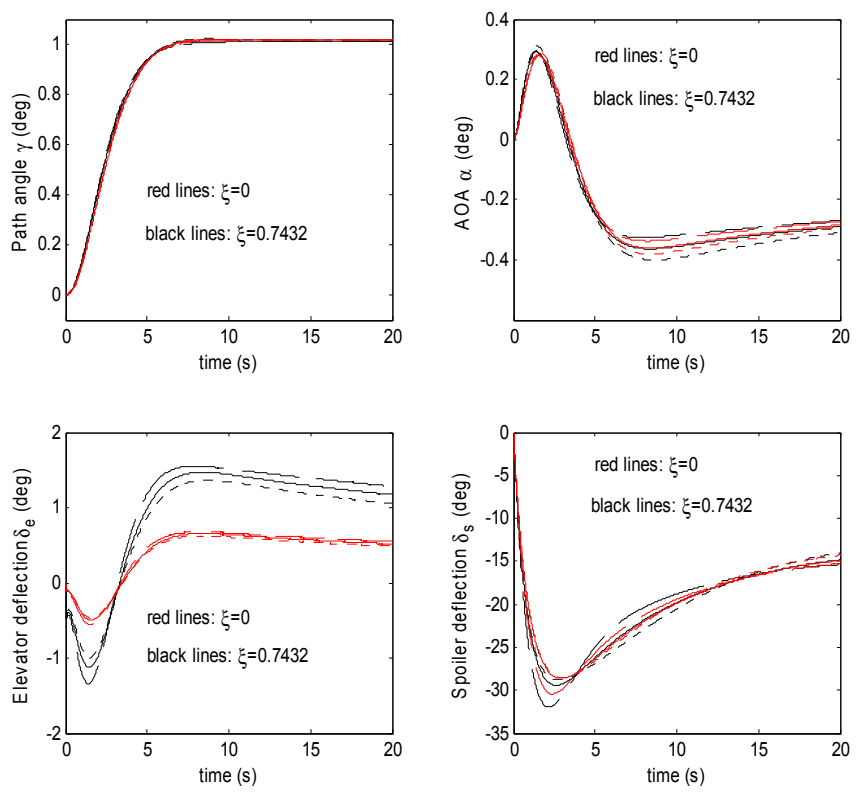

Fig. 3. Closed-loop responses of F-4 aircraft with $\xi=0$ and $\xi=0.7432$

\section{Conclusions}

This paper investigates a special simultaneous design optimization problem for integrated plant/outputfeedback controller where only one plant parameter has to be determined. By using the projection lemma Lemma 2, the optimization of plant parameter can be separated from the optimization of dynamic outputfeedback controller parameters. And both of them are based on solvability of LMIs. The F-4 fighter example demonstrates that the proposed approach is effective on the integrated plant/output-feedback controller optimization design. Next, this approach will be extended to handle the integrated plant/output-feedback controller optimization design where more than one plant parameters have to be determined.

\section{References}

[1] P. Apkarian, H. D. Tuan and J. Bernussou, "ContinuousTime Analysis, Eigenstructure Assignment, and $\mathrm{H}_{2}$ Synthesis With Enhanced Linear Matrix Inequalities (LMI) Characterizations", IEEE Trans. Automat. Contr., Vol. 46, pp. 1941-1946, 2001.

[2] E. Berglund and I. Kaminer, "An Integrated Approach to Plant Optimization and Controller Design," in Proc. IFAC Robust Control Design, Budapest, Hungary, pp. 213-218, 1997.

[3] H. K. Fathy, J. A. Reyer, P. Y. Papalambros and A. G. Ulsoy, "On the Coupling between the Plant and Controller Optimization Problems," in Proc. American Control Conference, Arlington, VA USA, pp. 1864-1869, 2001.

[4] K. M. Grigoriadis and F. Wu, "Integrated $H_{\infty}$ Plant/Controller Design via Linear Matrix Inequalities," in Proc. 36th IEEE Conference on Decision \& Control, San Diego, California USA, pp. 789-790, 1997.

[5] F. Liao, K. Y. Lum and J. L. Wang, "Mixed $H_{2} / H_{\infty}$ SubOptimization Approach for Integrated Aircraft/Controller Design" accepted for presenting in the 16th IFAC World Congress, Prague, Czech Republic, July, 2005.

[6] P. G. Maghami, S. M. Joshi and D. B. Price, "Integrated Controls-Structures Design Methodology for Flexible Spacecraft," Journal of Spacecraft and Rockets, Vol. 32, 1995.

[7] R. J. Niewoehner and I. I. Kaminer, "Integrated AircraftController Design Using Linear Matrix Inequalities," Journal of Guidance, Control and Dynamics, Vol. 19, No. 2, pp. 445$452,1996$.

[8] V. Sahasrabudhe, R. Celi and A. L. Tits, "Integrated RotorFlight Control System Optimization with Aeroelastic and Handling Qualities Constraints," Journal of Guidance, Control and Dynamics, Vol. 20, 1997.

[9] C. Scherer, P. Gahinet and M. Chilali, "Multiobjective Output-Feedback Control via LMI Optimization," IEEE Trans. on Automatic Control, Vol. 42, N0. 7, pp. 896-911, 1997.

[10] G. H. Yang and K. Y. Lum, "An optimization Approach to Integrated Aircraft-Controller Design," in Proc. American Control Conference, Denver, Colorado USA, pp. 1649-1654, 2003.

[11] K. Youcef-Toumi, "Modeling, Design and Control Integration: A Necessary Step in Mechatronics," IEEE/ASME Trans. on Mechatronics, Vol. 1, 1996.

[12] K. M. Zhou, J. C. Doyle, "Essentials of Robust Control", Prentice-Hall Int. Inc., 1998. 